
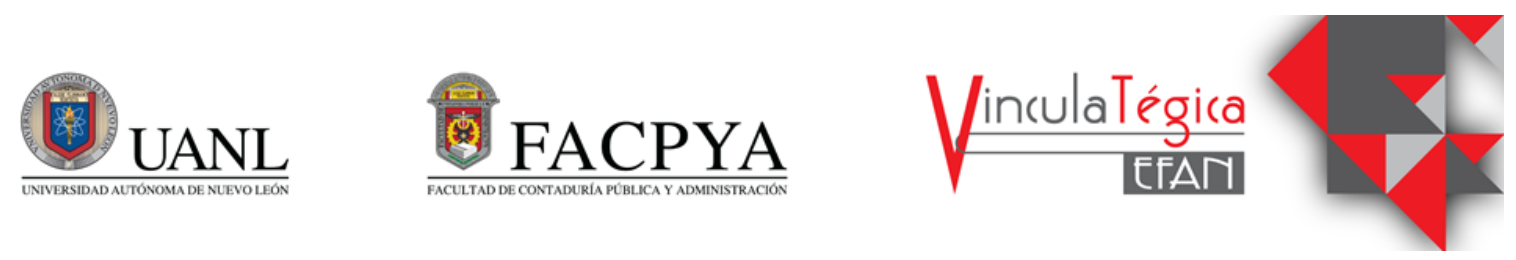

\title{
Digitalización en la auditoría externa-desafíos para la educación y la formación profesional en México
}

\author{
Manrico Maria Scheliga ${ }^{1}$ \\ ${ }^{1}$ Universidad Autónoma de Nuevo León, Facultad de Contaduría Pública y Administración, \\ mscheligax@uanl.edu.mx.com, San Nicolás de los Garza, N.L., +8117937325.
}

Información del artículo revisado por pares

Fecha de aceptación: junio-2021

Fecha de publicación en línea: diciembre-2021

DOI: https://doi.org/10.29105/vtga7.2-16

\section{Resumen}

La digitalización plantea importantes desafíos para los auditores externos y los auditores internos en particular. Para que las profesionales de la auditoria y la próxima generación de auditores estén preparadas para el futuro digital, es importante comprobar los conceptos de la formación universitaria y educación profesional en una etapa temprana y adaptarlos si es necesario. El siguiente artículo describe las posibles consecuencias de la digitalización para los profesionales de la auditoria externa e interna. Se entrevistó a de 108 auditores externos e internos para proporcionar una descripción general de los requisitos técnicos futuros para futuras carreras relacionadas a la auditoria. Los cambios necesarios en la educación universitaria, así como en la educación profesional continua, y se pueden derivarse de las respuestas.

Palabras clave: Auditoria externa, formación profesional, educación, digitalización, BIG 4, ecuaciones estructurales.

\section{Introducción}

Los crecientes cambios en las empresas debido a la digitalización también requieren la condición profesional de los auditores externos y auditores internos para adaptarse a nuevas condiciones como la Industria 4.0,

\begin{abstract}
Digitization poses significant challenges for external auditors and internal auditors in particular. For auditing professionals and the next generation of auditors to be prepared for the digital future, it is important to test the concepts of college and career education at an early stage and adapt them if necessary. The following article describes the possible consequences of digitization for external and internal audit professionals. 108 ecternal and internal auditors were interviewed to provide an overview of future technical requirements for future auditing-related careers. Changes needed in college education, as well as continuing professional education, and can be derived from the answers.
\end{abstract}

Keywords: external audit, vocational training, education, digitization, BIG 4, structural equations.

blockchain, cloud-computing, big data e inteligencia artificial. Las profesiones no solo tienen que desarrollarse más, sino que sus conceptos de educación universitaria y educación profesional también se enfrentan a nuevas oportunidades y desafíos para 
mantenerse al día con las innovaciones tecnológicas (Adiloglu \& Gungor, 2019). Con la educación profesional continua se refiere en el presente artículo a las capacitaciones que recibe el personal de auditoria por parte del despacho y forma parte de su currícula profesional dentro de la firma.

En este contexto, surge la pregunta de investigación de si la digitalización en la auditoría externa en México requiere una expansión de los conceptos de formación universitaria y educación profesional para incluir los conceptos básicos de la informática formal, estadísticas y análisis de modelos de negocio acorde de las sugerencias internacionales de educación. Para poder responder a la pregunta de investigación con una base en la evaluación de los practicantes de la profesión, se entrevistó a auditores externos de empresas auditoras mexicanas, empresas industriales y proveedores de servicios financieros.

\section{MARCO TEÓRICO}

En este capítulo se van a presentar los principales constructos de la digitalización de la auditoría externa en relación de la educación universitaria y formación profesional.

\subsection{Necesidad del cambio del aprendizaje universitario y profesional}

\subsubsection{Marco internacional y el cambio de la educación en la auditoría}

La profesión de contabilidad y auditoría opera en un entorno que está cambiando a un ritmo acelerado. Es responsabilidad de la profesión velar por que todos sus representantes cumplan con las expectativas que los usuarios de sus servicios tienen de ellos. Para que los contadores profesionales sigan siendo relevantes, es posible que deban cambiar o adaptar los servicios que ofrecen a sus clientes. Es responsabilidad de los organismos contables profesionales planificar estos cambios para garantizar que los miembros que se incorporen a la profesión posean los conocimientos y las habilidades necesarios para ser relevantes y seguir siendo relevantes (Wessels, 2008). Los resultados de la investigación muestran que, en el siglo XXI, la profesión de contador necesita un nuevo modelo que sea capaz de dar respuesta a los cambios tecnológicos y desarrollos en el proceso de digitalización y e-transformación para que la profesión de contador es más eficaz. Teniendo en cuenta los desarrollos y cambios tecnológicos, este modelado debería preparar la profesión de contador para el futuro reconstruyendo de punta a punta en campos como el pensamiento, la educación, la cultura y la tecnología (Tekbas \& Nonwoven, 2018). El papel de las instituciones de educación superior y las universidades en el desarrollo de la profesión de contador es igualmente importante. Hay muchas discusiones entre científicos y educadores sobre el futuro de la contabilidad y sobre las mejoras necesarias en los programas de estudio de los contadores para asegurar servicios contables valiosos y efectivos.

\section{Esto requerirá:}

a) una mayor comunicación entre las partes en términos del contenido y la pedagogía de los programas de aprendizaje desarrollados;

b) la transferencia de estrategias y experiencia para facilitar un enfoque estructurado para los futuros Nuevos Desafíos del Desarrollo Económico y Empresarial - 2019: Incentivos para el crecimiento económico sostenible 763 contadores; y

c) la creación de un entorno laboral que permita a los graduados aprovechar y transferir los conocimientos y habilidades adquiridos (Howieson, $\mathrm{y}$ otros, 2014).

La Federación Internacional de Contadores (IFAC) es la organización mundial de la profesión contable dedicada a servir al interés público fortaleciendo la profesión y contribuyendo al desarrollo de economías internacionales fuertes. La IFAC está compuesta por más de 175 miembros y asociados en más de 130 países y jurisdicciones, que representan a casi 3 millones de contadores en la práctica pública, la educación, los servicios gubernamentales, la industria y el comercio. La visión de IFAC es que la profesión global del contador sea 
reconocida como esencial para organizaciones, mercados financieros $y$ economías fuertes y sostenibles. El Plan Estratégico de IFAC, "Trazando el futuro de la profesión global", identifica oportunidades y riesgos para la profesión dentro del contexto del entorno actual y anticipado, al tiempo que destaca la posición única y los beneficios económicos de las actividades de IFAC, así como actividades planificadas de IFAC en respuesta a macrotendencias con implicaciones significativas para la profesión (IFAC , 2018). Con el fin de asegurar la realización de los objetivos estratégicos, se han establecido varios consejos y comités en la IFAC que trabajan con los problemas educativos y de desarrollo de los contadores: Junta Internacional de Normas de Educación Contable (IAESB) y Comité de Desarrollo de la Organización de Contabilidad Profesional (PAO). La tarea del Comité de Desarrollo PAO de la IFAC es ayudar a la IFAC a lograr organizaciones profesionales de contadores sólidas y sostenibles en todo el mundo, la fuente más eficaz, eficiente y sostenible para promover la profesión de contador. El comité actúa como un importante asesor estratégico de IFAC, contribuyendo activamente a los objetivos estratégicos de IFAC y ayudando con la implementación. Esto incluye aumentar la comprensión e identificar soluciones a los desafíos que enfrenta el desarrollo de PAO sólidas, así como promover, establecer y fortalecer las PAO en apoyo del interés público (IFAC , 2018). IAESB está trabajando desde 1977 y el resultado del trabajo de esta organización son los Estándares Internacionales de la educación de los contadores (IES) que se revisan de vez en cuando para implementar correcciones de acuerdo con la situación económica cambiante. El borrador de los últimos cambios fue preparado y publicado para discusiones en 2015. IAESB ha publicado un Documento de Consulta, "Cumplir con las expectativas futuras de competencia profesional: una consulta sobre la estrategia y prioridades futuras del IAESB". Presenta la visión y la estrategia propuestas para los próximos cinco años que se basan en la finalización de sus Estándares Internacionales de Educación recientemente revisados y su trabajo para apoyar la implementación de estos estándares. Al emitir este Documento de Consulta, el IAESB reconoce que los contadores profesionales están operando en un entorno que cambia continuamente. Esto tiene un impacto en las necesidades iniciales y continuas de desarrollo profesional de los contadores profesionales y las demandas que se les imponen a los contadores profesionales a nivel mundial (IAESB, 2015). Al contemplar la mejor manera de responder al entorno cambiante de la educación contable y la necesidad de proteger el interés público, el IAESB prevé sus actividades prioritarias de la siguiente manera: mejorar los estándares existentes, desarrollar nuevos estándares para llenar los vacíos identificados en la educación contable profesional, preparar material para respaldar adopción e implementación de las IES y facilitación del liderazgo intelectual en temas relevantes. Se alentó especialmente la retroalimentación de los organismos miembros de la IFAC en todas las jurisdicciones; partes interesadas en políticas públicas, regulación, contabilidad pública y otras comunidades relevantes; contadores profesionales en el ámbito empresarial, gubernamental y académico; y empleadores de contadores profesionales (IFAC , 2018). Los organismos miembros de la IFAC revisarán y actualizarán periódicamente los programas de educación profesional en contabilidad que están diseñados para lograr los resultados de aprendizaje establecidos en esta IES (IAESB, 2015).

\subsubsection{Educación universitaria clásica}

La educación universitaria tradicional en economía en los campos de auditoría externa incluye, en el sentido más estricto, clases y seminarios sobre auditoría empresarial, contabilidad y derecho comercial, incluido el derecho tributario. En un sentido más amplio, también se imparten clases y seminarios sobre el gobierno corporativo, valoración de empresas, financiación, contabilidad de costes y economía. Además, la mayoría de los reglamentos de estudio y los reglamentos de exámenes en el programa de licenciatura en 
economía prevén conferencias obligatorias sobre matemáticas, incluida la estadística. Las clases o seminarios obligatorios sobre informática que también se ofrecen en la universidad, sin embargo, son generalmente más una descripción general. En las materias de especialización de auditoría y auditoría interna, las conferencias sobre informática y estadística son actualmente la excepción predominante (Gulin, Hladika, \& Valenta, 2019).

Del mismo modo, las áreas de examen en el examen de auditoría empresarial hasta ahora no han mostrado ningún requisito específico que haga justicia a los desarrollos descritos en la introducción (Plant, Barac, \& Sarens, 2019). Por otro lado, las especificaciones para el Examen de Auditor Interno Certificado establecen al menos algunos requisitos del campo del análisis de datos basados en TI y técnicas de evaluación como la minería de datos (Barrados \& Lonsdale, 2020).

\subsubsection{Razones del cambio de la perspectiva académica}

Desde un punto de vista académico, hay dos razones principales para esta falta de clases y seminarios sobre tecnología de la información. En primer lugar, cuantas que más clases y seminarios de informática contenga, más difícil será distinguir una licenciatura en economía, contabilidad y administración de una licenciatura en informática o informática empresarial. Para preservar el perfil más nítido posible de las carreras de economía, contabilidad y administración, siempre ha habido reservas por parte de las facultades en el pasado para integrar las clases o seminarios de tecnología de la información en las carreras de las escuelas de negocios (Springer Sargent \& Faye Borthick, 2013).

En segundo lugar, las ciencias económicas están naturalmente posicionadas de manera amplia debido a sus componentes comerciales y económicos. Por tanto, en el pasado, no era necesario ampliar este espectro con conferencias de informática. Por cierto, esto también se aplica a los cursos puramente de gestión empresarial, ya que sus subáreas, como contabilidad, derecho fiscal, control o financiación, se han vuelto tan complejas y de múltiples niveles que pueden justificar sus propias licenciaturas (Vasarhelyi, Teeter, \& Krahel, 2010).

Según la opinión aquí representada, es deseable, pero no absolutamente necesario, un conocimiento profundo de la informática, que también incluye el manejo profesional de lenguajes de programación específicos. Más bien, las últimas décadas han demostrado que los conceptos de uso se están volviendo cada vez más intuitivos. Si bien los primeros sistemas operativos solo podían controlarse con líneas de comando, por ejemplo, los conceptos de control visual, háptico y verbal están muy extendidos en la actualidad. Además, los servicios especializados podrían asumir tareas técnicamente exigentes en el campo de las TI (Timea Fulop, Tiron-Tudor, \& Cordos, 2019). Además, la forma algorítmica de pensar que transmite el conocimiento básico de la informática no debería resultar tan ajena a los estudiantes del campo de la auditoría como parece a primera vista. Finalmente, algunas normas contables y normas de auditoría siguen un esquema de prerrequisito y consecuencia legal, que corresponde al principio si-entonces de un algoritmo (Pedrosa \& Costa, 2012).

\subsubsection{Causas desde la perspectiva del mercado laboral}

Además de las razones académicas descritas, la falta de clases y seminarios sobre tecnología de la información en las áreas de auditoría externa a menudo puede justificarse por el trabajo diario de los auditores externos $\mathrm{o}$ auditores internos. El equipo respectivo cuenta con el apoyo de un auditor de TI para que el auditor o auditor interno pueda concentrarse en temas que son esenciales y propensos a errores por razones de capacidad y eficiencia (Stoel, Havelka, \& Merhout, 2012). Para poder evaluar esto, el auditor o auditor interno debe tener un conocimiento básico de los algoritmos, heurísticas y modelos estadísticos utilizados (Henrizi, Himmlesbach, \& Hunziker, 2021). Por lo tanto, los conceptos de capacitación en las áreas de auditoría debe incluir al menos los conceptos básicos de TI y estadísticas 
formales (Maradona, 2020). Otra razón de la falta de clases y seminarios de TI surge del perfil de requisitos del mercado laboral para los jóvenes profesionales en auditoría externa y auditoría interna. En las últimas décadas, normalmente era suficiente para una carrera en la auditoría si los graduados había cursado las clases y seminarios antes mencionadas sobre auditoría con buenas calificaciones y tenían suficiente experiencia en el manejo de software para poder trabajar después un breve período de formación para dominar las herramientas y técnicas de auditar con soporte de TI del empleador (Manita, Elommal, Baudier, \& Hikkerova, 2020).

\subsection{Impacto de la digitalización}

La digitalización acelerada de la economía pone en tela de juicio el abandono previo de las conferencias de TI en las áreas especializadas de auditoría (Adiloglu \& Gungor, 2019). Antes de adaptar los conceptos de formación y educación superior, se debe discutir la cuestión de qué efectos específicos podría tener la digitalización en la auditoría externa (Cipek \& Ljutić, 2021). Aquí son concebibles al menos dos escenarios.

Gartner (2020) define la digitalización como el uso de tecnologías digitales para cambiar un modelo de negocio y proporcionar nuevos ingresos $\mathrm{y}$ oportunidades de producción de valor; es el proceso de pasar a un negocio digital. Los primeros rastros de digitalización se desarrollaron durante la Revolución Industrial cuando nuevas máquinas cambiaron el proceso del comercio, el capitalismo y, de hecho, la historia humana (Westerman, Bonnet y McAfee, 2014). Como en el presente, la innovación en herramientas digitales está llevando al mundo y sus habitantes a la segunda era de las máquinas, como Brynjolfsson y McAfee (2014) definieron en su libro del mismo título. Que hoy día se conoce bajo el término de la industria 4.0. Zuboff (1988) utiliza el término digitalización para referirse a las innovaciones tecnológicas que generan oportunidades, lo que posteriormente crea un mundo contemporáneo para los individuos. En cuanto al nivel superior, la digitalización incluye las transformaciones masivas que ocurren en la sociedad y las industrias con la ayuda de las tecnologías digitales (Majchrzak et al., 2016).

\subsubsection{Automatización de actividades rutinarias}

En el primer escenario, la digitalización da como resultado una amplia automatización de las tareas rutinarias para que el auditor externo o auditor interno pueda concentrarse en evaluar casos especiales (Abbott, Parker, Peters, \& Rama, 2007).

\subsubsection{Automatización de actividades rutinarias}

En el segundo escenario, los algoritmos y heurísticos altamente desarrollados, en lo sucesivo denominados automatismos, también asumen tareas exigentes para las que se requiere juicio. Como consecuencia, el auditor externo $o$ el auditor interno serían prescindibles en muchas áreas de la auditoría tradicional. En la economía del comportamiento, el automatismo es el término utilizado para describir todas las actividades que se llevan a cabo sin ninguna guía y control reconocibles, voluntarios o externos. Esto los distingue de los reflejos y el comportamiento instintivo, que al menos pueden estar influenciados por factores externos. (Tiberius \& Hirth, 2019).

\subsubsection{Automatización de actividades rutinarias}

En caso de que los automatismos asuman realmente tareas exigentes en materia de auditoría y auditoría interna, la baja necesidad de auditores o auditores internos podría compensarse, al menos parcialmente, con la disminución de los jóvenes profesionales en los últimos años (Tiberius \& Hirth, 2019). Desde el punto de vista de la profesión y los profesores universitarios en estos campos, es probable que este desarrollo represente más un desafío que una solución de problema, por lo que no se analizará más aquí. A continuación, se examinan los límites técnicos de los automatismos que hablan en contra del subempleo de los auditores o auditores internos debido a la digitalización.

Hasta ahora, incluso los automatismos altamente desarrollados han dado resultados incorrectos en situaciones imprevistas. El 
hecho de que los imprevistos sean más la regla que la excepción en servicios complejos habla en contra del uso de automatismos para tareas exigentes en la auditoría. Además, los automatismos pueden manipularse mediante entradas incorrectas deliberadas, por ejemplo, en el caso de tipos de datos incompatibles, o mediante entradas incorrectas deliberadas, por ejemplo, por hackers informáticos.

Una solución sensata podría ser el uso de automatismos en combinación con un control final por parte del auditor o auditor interno. Adiloglu \& Gungor (2019) dicen que es improbable que el auditor externo o el auditor interno sean prescindibles en tales tareas en el futuro previsible, ya que incluso los automatismos altamente desarrollados en el campo de la inteligencia artificial no son capaces de abstracción o creatividad humana. Además, incluso el software altamente desarrollado debe ser manejado por humanos que, por ejemplo, la existencia de varias facturas con el mismo número de factura es un signo de irregularidades en la contabilidad o que el riesgo de medidas de modelación de hechos es particularmente alto si las expectativas del analista no se cumplen.

En otras palabras, la digitalización obviamente puede hacer prescindible al auditor o auditor interno en algunas tareas, en otras lo exige aún más como experto. Esto es particularmente evidente en desarrollos como la Cloud Computing, la Industria 4.0 o en la adquisición y evaluación de big data. El aumento asociado en la complejidad de los modelos comerciales y su aparición y destrucción aceleradas presentan al auditor y al auditor interno desafíos completamente nuevos (Balios, 2021). De acuerdo con la opinión aquí representada, los conceptos de formación en los campos de auditoría externa debería, por tanto, ampliarse para incluir el análisis de modelos de negocio, especialmente digitales, además de los conceptos básicos de TI y estadísticas (Barrados \& Lonsdale, 2020).

\subsection{Tendencias en la digitalización de la auditoria}

En lo siguiente se van a presentar las tendencias más significativas de la digitalización en la auditoria:
Blockchain es un tipo de tecnología de contabilidad distribuida (DLT) que aún se encuentra en una etapa inicial, pero hasta ahora muestra una gran promesa de cambiar radicalmente la forma en que las organizaciones llevan a cabo sus negocios y, por lo tanto, cómo son auditadas (Schulman \& Wilson, 2019). Blockchain podría describirse como un libro de contabilidad digital descentralizado que registra las transacciones entre dos partes en una red de igual a igual (P2P) y las cifra (Narayanan, Bonneau, Felten, Miller, \& Goldfeder, 2016). Según Vaidyanathan (2017), DLT se asegura de que "en un libro mayor distribuido, todos los participantes tengan una visión común de los registros". El mismo autor afirma además que esto es lo contrario de la situación típica actual en la que los participantes pueden estar mirando diferentes bases de datos que se actualizan y editan por separado (Vaidyanathan, 2017). Además, una red blockchain se puede dividir en dos categorías: pública y privada. Una red pública es aquella a la que cualquiera puede unirse libremente ya que no existe una autoridad central, esto también se llama red sin permiso. Una privada es cuando solo los usuarios autorizados tienen derechos de lectura y escritura (Narayanan, Bonneau, Felten, Miller, \& Goldfeder, 2016), también llamada red autorizada.

Sin duda, la inteligencia artificial es uno de los inventos humanos más prometedores y al mismo tiempo intimidantes de los últimos años según el fundador de Microsoft, Bill Gates (Clifford, 2019). La inteligencia artificial, y en ocasiones también conocida como inteligencia de máquina, se considera la inteligencia exhibida por las máquinas en comparación con la inteligencia heredada de humanos y animales. Se puede decir que la inteligencia artificial es un término genérico para las tecnologías que pueden usarse por sí mismas o en combinación entre sí para imitar comportamientos cognitivos que se comparten entre los humanos, como el aprendizaje y la resolución de problemas (Issa, Sun, \& Vasarhelyi, 2016). Estas tecnologías de relevancia para la auditoría son: procesamiento del lenguaje natural 
(NLP), aprendizaje profundo (DL) y aprendizaje automático (ML). La $P N L$ se refiere a la capacidad de las computadoras para comprender el lenguaje humano tanto en texto como en voz. Por lo general, después de la $P N L$, se aplica el aprendizaje automático para extraer información útil (Yordanov, 2018). El mayor beneficio de la $P N L$ en la auditoría es la velocidad. La $P N L$ puede examinar cientos de miles de documentos a una velocidad incontestable en comparación con los humanos.

Big data es responsable de conjuntos de datos cuyo volumen excede la capacidad de las técnicas de software de base de datos promedio para capturar, almacenar, administrar y analizar. Meuldijk (2017) define la necesidad de big data para los auditores como una herramienta significativa para impulsar la evaluación de riesgos, el alcance, el análisis de tendencias y los juicios. De acuerdo con la investigación analítica actual (Howieson, y otros, 2014), Big Data tiene que ofrecer a los auditores la posibilidad de operar análisis prescriptivos como implementar prácticas que verifican computacionalmente las acciones existentes y sus resultados, con respecto a ciertas dificultades, reglas $\mathrm{y}$ limitaciones (Lee, Cho, Gim, Jeong, \& Jung, 2014). Dado que existe una evaluación actual a través de la auditoría que ha enfrentado varios tipos de fallas en los últimos años, por ejemplo, Enron y WorldCom a la crisis financiera (ICAEW 2010; ACCA 2011), se recomienda que los auditores involucren con entusiasmo a Big Data como una estrategia para mejorar el éxito y la integridad de sus productos y servicios organizacionales (Alles, 2015). De ahí que los auditores posiblemente puedan definir Big Data como una herramienta para eliminar los gastos de sus auditorías y mejorar la rentabilidad en el caso de los auditores externos y la rentabilidad para los auditores internos que son responsables de (Littley, 2012). Además, uno de los usos más implícitos del análisis de Big data por parte de los profesionales de la auditoría podría ser la mejora de las visiones con respecto a las transacciones comerciales con datos externos, no contables, originados a partir de Big data que se pueden complementar con datos contables para la credibilidad, como los no contables. la información derivada de las redes sociales se puede utilizar en transacciones o valoraciones de activos (Yoon, Hoogduin, \& Zhang, 2015). Sin embargo, con respecto a la naturaleza de Big Data, con sus diversas fuentes de datos que pueden ser diferentes o inusuales para que los auditores las utilicen en la investigación, se requiere que los patrones en el contexto de las fuentes de datos de auditoría sean revisados y posiblemente re-investigados en consideración de Big Data. A pesar de la fuente, los datos deben ser fiables y válidos.

Cloud Computing es una nueva forma de subcontratación de sistemas e infraestructura de TI como alternativa a la subcontratación de TI tradicional (ITO). Por lo tanto, la migración a la computación en la nube está creciendo rápidamente entre las organizaciones. La adopción de esta tecnología aporta numerosos aspectos positivos, aunque impone diferentes riesgos e inquietudes a la organización. Una organización que delega oficialmente sus servicios de computación en la nube a proveedores externos (offshore o inshore) e implica que subcontrata sus funciones $\mathrm{y}$ procesos de su TI a proveedores externos de servicios de subcontratación de procesos comerciales. Por lo tanto, los clientes de la nube deben evaluar y administrar la construcción de la infraestructura de TI y el entorno de control de TI de la organización de los proveedores de subcontratación de procesos comerciales. Dado que la nube es una tecnología basada en Internet, la auditoría de la nube sería muy crítica y desafiante en un entorno de este tipo.

\section{MÉTODO}

\subsection{Estrategia de investigación}

Recker (2013) describe la selección de la metodología de investigación como una acción vital en el proceso de investigación que también puede definirse como la estrategia de indagación utilizada para investigar un problema de investigación en particular. Dado que la investigación tiene como objetivo un análisis exhaustivo de los efectos de la digitalización en la parte educativa de 
auditores, es importante que la estrategia de investigación aplicada pueda contribuir significativamente a cumplir con éxito estos objetivos de investigación. Esta investigación utiliza principalmente métodos de investigación cualitativos, ya que se recomiendan como adecuados para el procesamiento de datos no numéricos con el fin de obtener una comprensión profunda de los fenómenos sociales, como diferentes perspectivas, significados y conceptos (Bhattacherjee, 2012). Como las opiniones o justificaciones de los individuos detrás de sus acciones están muy vinculadas con el significado contextual, los métodos de investigación cualitativa son la opción adecuada para descubrir este mismo contexto para proporcionar aclaraciones lógicas sobre las razones detrás del desarrollo de los fenómenos (Recker, 2013). La investigación cualitativa cubre significados contextuales como los comportamientos, opiniones y experiencias de las personas (Mohajan, 2018). Para fundamentar teóricamente este estudio, se ha realizado de antemano una revisión de la literatura académica sobre el tema de la digitalización y la auditoría. Los conceptos más importantes se mencionan en el marco teórico del presente trabajo. De esta revisión se encontró que la literatura no existe un estudio en México completamente cubierta en términos de esta área específica donde se recaba la opinión de los profesionales en los despachos contables de los BIG 4 en México. En consecuencia, se adopta una estrategia cualitativa para el estudio de los fenómenos que no han sido descubiertos de manera integral (Recker, 2013). Por lo tanto, Recker (2013) y Bhattacherjee (2012) sugieren que los métodos cualitativos son la estrategia recomendada en términos de estudiar el tema razonablemente poco estudiado como los efectos de la digitalización en la educación de la profesión de auditoría en México.

Para el estudio se desarrolla un cuestionario para recabar las opiniones de los profesionales y académicos para los impactos de la digitalización en la educación para los auditores externos e internos.

\subsection{Estrategia de investigación}

Para realizar una correcta distribución del sector de los auditores externos de los despachos contables nacionales (población) que auditan empresas que cotizan en la bolsa mexicana de valores (BMV), la muestra de despachos contables a analizar será determinada utilizando muestreo aleatorio estratificado, considerando que este es aplicado con el fin de garantizar que cada grupo de la población se encuentre representado en la muestra (Lind, Marchal, \& Wathen, 2012).

Para la población se identifica que en México hay en total 116 clusters de los Big 4 que auditan las empresas que cotizan en la bolsa mexicana. Estos 116 clusters representan todos los equipos de auditoria que dictaminan de la información de las empresas públicas. Para la encuesta se va a entrevistar mediante un cuestionario en google forms un representante de auditoría de cada cluster. Su puesto puede se socio de auditoria o gerente de un equipo de auditoria que tiene una empresa publica en su cartera de clientes.

Considerando que el estudio a realizar para determinar la muestra de la población es finita y que la variable principal el cambio de la educación en la auditoría por el impacto de la digitalización es cualitativa, se utiliza la siguiente ecuación para calcular el tamaño de la muestra de la proporción de una población finita:

Ecuación 1- Cálculo de la muestra para una población finita $\mathrm{N}$

$$
n=\frac{N P Q}{(N-1) * \frac{e^{2}}{Z}+P Q}
$$

Donde:

$\mathrm{N}=$ tamaño de la población
$\mathrm{n}=$ tamaño de la muestra
$\mathrm{P}=$ proporción del evento de interés
$\mathrm{Q}=$ complemento de $\mathrm{P}$
$\mathrm{e}=$ error tolerado en porcentaje estimado

El cálculo del tamaño de la muestra basado en la formula anterior se precisó que esta debe incluir 51 representantes de socios o gerentes que llevan a cabo las auditorias de las empresas que cotizan en bolsa. La población total son 116, esto con un nivel de confianza del estadístico $\mathrm{Z}$ de $95 \%$ y un error de estimación máximo aceptado del $5 \%$, esto se 
represente en la siguiente ecuación:

Ecuación 2- Cálculo de la muestra de N $=116$

$50.82=\frac{(116) *(1.96)^{2} *(0.5) *(0.5)}{\left(0.05^{2} *(116-1)+(1.96)^{2} *(0.5) *(0.5)\right.}$

En general, la evaluación tiene en cuenta las respuestas de 108 auditores externos. Cada cuestionario representa una empresa de auditoría o una empresa. La mayoría de los auditores encuestados $(80.6 \%)$ trabaja para una empresa de auditoría más grande (BIG 4) de los despachos en México, Guadalajara y Monterrey. El residual del $19.4 \%$ se coloca en despachos de los Big 4 más pequeños de la república mexicana. En estos despachos el servicio de la auditoria anual es un área central de actividad. Los auditores con un enfoque en el ámbito educativo con puesto en universidades en México representan $11.1 \%$ de los encuestados.

Los encuestados indican su nivel de aprobación o rechazo marcando una de las opciones Es completamente correcto, Estoy de acuerdo, No Estoy de acuerdo, Estoy totalmente en desacuerdo o No lo puedo estimar. La última opción de respuesta en la evaluación es la misma en la evaluación con valores perdidos. La encuesta se compartió con la plataforma de Google Forms y fue contestado durante los meses abril y mayo del 2021.

\subsection{Hipótesis}

En esta sección, se discute en detalle las hipótesis que subyacen al modelo:

H1: La digitalización en la auditoria tiene un impacto a las clases y seminarios en estudios de economía, contabilidad y administración empresas.

H2: La cantidad de la información generada por la digitalización tiene un impacto a las clases y seminarios en estudios de economía, contabilidad y administración empresas.

H3: La digitalización tiene impacto en la planeación de recursos humanos en la auditoria.

H4: La digitalización impacta el plan de estudios de auditoría en las universidades y en la formación profesional en las empresas.

H5: Las nuevas áreas de estudio de la digitalización tienen un impacto al plan de estudios de auditoría en las universidades y en la formación profesional en las empresas.

H6: Las nuevas áreas de estudio de la digitalización tienen un impacto en las clases y seminarios de la auditoría en la universidad y formación profesional en las empresas.

H7: La cantidad de información generada por la digitalización tiene impacto a las nuevas áreas de estudio.

H8: La planeación de recursos humanos en la era de la digitalización de la auditoría tiene un impacto a las clases y seminarios en la universidad y la formación profesional.

H9: Las nuevas áreas de estudio tienen un impacto a la digitalización de la auditoria.

H10: La cantidad de nueva información en la auditoria tiene un impacto a la digitalización la auditoría.

\subsection{Análisis de datos}

La técnica elegida para testear el modelo es SEM, que permite emplear adecuadamente variables latentes y proporciona una mejor comprensión de sus relaciones. La técnica tiene dos etapas. El primero es el modelo de medición, que especifica qué tan bien se miden los constructos en términos de las variables observadas. El segundo es el modelo estructural, que se centra en las relaciones entre constructos. Se ha estimado mediante Partial Least Square (PLS) utilizando el software PLS-Graph (Chin, 2001).

La primera etapa en el análisis PLS es el modelo de medición, que se basa en los cálculos del Análisis de componentes principales (ACP). Aunque ACP es una técnica exploratoria comúnmente utilizada en la investigación contable, la forma en que opera en SEM es diferente. Esto se debe a que SEM adopta un enfoque deductivo. Los investigadores comienzan proponiendo los indicadores en el constructo; los que no cumplen con ciertos atributos, como unidimensionalidad, confiabilidad, validez convergente y validez discriminante, son posteriormente rechazados.

Para probar la unidimensionalidad, se realizaron análisis de PCA en todos los indicadores para todos los constructos del 
estudio. En total, se realizaron análisis de PCA: uno para cada constructo. Luego se aplicó el criterio de (Kaiser, 1960): obtener un valor propio mayor que 1 solo en el primer componente principal.

La confiabilidad evalúa la consistencia de los indicadores en el constructo (todos los indicadores deben medir lo mismo). Los investigadores calcularon el alfa de Cronbach (Cronbach, 1970) y la fiabilidad compuesta (Werts, Linn, \& Jöreskog, 1974), clasificando los indicadores de 0 (ausencia de homogeneidad) a 1 (máxima homogeneidad). La diferencia entre los dos cálculos es que el alfa de Cronbach presupone que cada indicador en un constructo tiene el mismo peso, mientras que la confiabilidad compuesta usa pesos de ítem del modelo causal. El criterio de fiabilidad habitual es que ambos índices alcancen valores superiores a 0.7. La grafica 2 muestra los valores alfa de Cronbach. Todos los constructos superan el valor recomendado de 0.7 .

\section{RESULTADOS}

Para el análisis se probó la importancia de todas las rutas del modelo estructural utilizando un procedimiento de remuestreo de arranque con 500 iteraciones. La Tabla 3 muestra los coeficientes de regresión estandarizados $\beta$ denominados coeficientes de trayectoria en la jerga SEM. También se muestra los valores t de Student y R-cuadrado, que se interpretan de la misma forma que las regresiones convencionales.

Los valores de R-cuadrado miden la varianza de constructo explicada por el modelo. El R-cuadrado de "clases y seminarios enfocados en digitalización", la variable latente a explicar, es 0.533. Los valores de $\mathrm{R}$ cuadrado para el resto de las variables latentes son 0.401 para "Digitalización en la auditoría", 0.237 para "Planes de estudio de las universidades / formación profesional", 0.212 para "Recursos humanos" y 0.115 para "Nuevas Áreas de estudio".

Los coeficientes de trayectoria estandarizados nos permiten analizar el grado de cumplimiento de las hipótesis. Chin (1998) sugiere que deberían ser superiores a 0.3 para ser considerados significativos. Falk \& Miller (1992) recomiendan multiplicar el "coeficiente de trayectoria" ( $\beta$ ) por el coeficiente de correlación entre variables latentes con el argumento de que estos datos son una medida razonable de la varianza del constructo explicada por la variable predictiva latente.

La hipótesis 1 establece que La digitalización en la auditoria tiene un impacto a las clases y seminarios en estudios de economía, contabilidad y administración empresas. La tabla 3 muestra un valor $\beta$ de 0.149 y un valor $\mathrm{t}$ de 1.402 , que es estadísticamente no significativo. $\mathrm{Al}$ multiplicar el valor de $\beta$ por el coeficiente de correlación entre ambas variables latentes (0.535) que es positivo y estadísticamente significativo, se obtiene 0.079715. Ésta es una medida no razonable de la varianza del constructo "clases y seminarios enfocados en digitalización" explicada por la variable predictiva latente "Digitalización en la auditoría". A partir de los valores de $\beta$, t-valor y R-cuadrado, se puede concluir que los datos rechazan la Hipótesis 1 (H1).

La hipótesis 2 relaciona La cantidad de la información generada por la digitalización tiene un impacto a las clases y seminarios en estudios de economía, contabilidad y administración empresas. El coeficiente de correlación entre ellos es de 0.416 (Tabla 2), el cual es positivo y estadísticamente significativo. Pero los valores de $\beta$ (0.180) y t-valor (1.923) son muy bajos. Como resultado, se rechaza la Hipótesis 2 (H2).

La hipótesis 3 relaciona la digitalización tiene impacto en la planeación de recursos humanos en la auditoria. El coeficiente de correlación entre ellos es de 0.460 (Tabla 2), el cual es positivo y estadísticamente significativo. Los valores de $\beta(0.460)$ y t-valor (5.065) son altos y arriba del 0.3. Como resultado, se aprueba la hipótesis 3 (H3).

La hipótesis 4 relaciona la digitalización impacta el plan de estudios de auditoría en las universidades y en la formación profesional en las empresas. El coeficiente de correlación entre ellos es de 
0.344 (Tabla 2), el cual es positivo per estadísticamente no significativo. Los valores de $\beta(0.264)$ y t-valor (2.158) son bajos. Como resultado, se rechaza la hipótesis 4 (H4).

La hipótesis 5 relaciona las nuevas áreas de estudio de la digitalización tienen un impacto al plan de estudios de auditoría en las universidades y en la formación profesional en las empresas. El coeficiente de correlación entre ellos es de 0.440 (Tabla 2), el cual es positivo y estadísticamente significativo. Pero los valores de $\beta(0.276)$ y t-valor (2.369) son muy bajos. Como resultado, se rechaza la Hipótesis 5 (H5).

La hipótesis 6 establece que las nuevas áreas de estudio de la digitalización tienen un impacto en las clases y seminarios de la auditoría en la universidad y formación profesional en las empresas. La tabla 3 muestra un valor $\beta$ de 0.564 y un valor $t$ de 4.161, que es estadísticamente no significativo. Al multiplicar el valor de $\beta$ por el coeficiente de correlación entre ambas variables latentes (0.696) que es positivo y estadísticamente significativo, se obtiene 0.3925 . Ésta es una medida razonable de la varianza del constructo "nuevas áreas de estudio de la digitalización" explicada por la variable predictiva latente "clases y seminarios de la auditoría en la universidad y formación profesional en las empresas". A partir de los valores de $\beta$, t-valor y R-cuadrado, se puede concluir que los datos apoyan la Hipótesis 6 (H6).

La hipótesis 7 relaciona la cantidad de información generada por la digitalización tiene impacto a las nuevas áreas de estudio. El coeficiente de correlación entre ellos es de 0.340 (Tabla 3), el cual es positivo y estadísticamente significativo. Los valores de $\beta(0.340)$ y t-valor (3.562) son altos y arriba del 0.3. Como resultado, se aprueba la hipótesis 7 (H7).

La hipótesis 8 relaciona La planeación de recursos humanos en la era de la digitalización de la auditoría tiene un impacto a las clases y seminarios en la universidad y la formación profesional. El coeficiente de correlación entre ellos es de 0.290 (Tabla 2), el cual es estadísticamente no significativo. Los valores de $\beta(-0.050)$ y t-valor (0.319) son bajos. Como resultado, se rechaza la hipótesis $8(\mathrm{H} 8)$.

La hipótesis 9 relaciona las nuevas áreas de estudio tienen un impacto a la digitalización de la auditoria. El coeficiente de correlación entre ellos es de 0.622 (Tabla 2), el cual es positivo y estadísticamente significativo. Los valores de $\beta(0.580)$ y tvalor (9.892) son altos y arriba del 0.3. Como resultado, se aprueba la hipótesis 9 (H9).

La hipótesis 10 la cantidad de nueva información en la auditoria tiene un impacto a la digitalización la auditoría. El coeficiente de correlación entre ellos es de 0.321 (Tabla 2), el cual es estadísticamente no significativo. Los valores de $\beta(0.124)$ y t-valor (1.520) son bajos. Como resultado, se rechaza la hipótesis 10 (H10).

\section{CONCLUSIONES}

El objetivo de esta investigación era obtener una evaluación de los profesionales sobre los efectos del aumento de la digitalización en la auditoría y los cambios resultantes para la formación y la educación superior. En general, se puede observar que hay un impacto significativo en la relación entre los impactos de la digitalización en la auditoría y la necesidad de crear un nuevo ambiente educativo para enfrentar todos los retos para que también las auditorias están preparadas para la industria 4.0.

En primer lugar, se afirme que la digitalización en el ámbito de la auditoría tiene impacto en la planeación de recursos humanos en la auditoria (H3). Por nuevas tecnologías, procesos de análisis y nuevas herramientas se puede reemplazar ciertas actividades rutinarias con el fin que el perfil de auditor cambiará y su educación universitaria y profesional. Esto se confirma en los hallazgos de Abbott, Parker, Peters, \& Rama (2007).

También, las nuevas áreas de estudio de la digitalización tienen un impacto en las clases y seminarios de la auditoría en la universidad y formación profesional en las empresas (H6) y la relación de las nuevas áreas de estudio tienen un impacto a la digitalización de la auditoria (H9). La digitalización obviamente puede hacer prescindible al auditor en algunas tareas, en otras lo exige aún 
más como experto. Esto es particularmente evidente en desarrollos como la Cloud Computing, la Industria 4.0 o en la adquisición y evaluación de big data. El aumento asociado en la complejidad de los modelos comerciales y su aparición y destrucción aceleradas presentan al auditor desafíos completamente nuevos (Balios, 2021). De acuerdo con aquí representada, los conceptos de formación en los campos de auditoría deberían, por tanto, ampliarse para incluir el análisis de modelos de negocio, especialmente digitales, además de los conceptos básicos de TI y estadísticas (Barrados \& Lonsdale, 2020).

La relación de la cantidad de información generada por la digitalización tiene impacto a las nuevas áreas de estudio (H7). Con la aprobación de esta hipótesis se confirma la necesidad de poder evaluar grandes cantidades de información, el auditor debe tener un conocimiento básico de los algoritmos, heurísticas y modelos estadísticos utilizados para analizar grandes cantidades de datos (Henrizi, Himmlesbach, \& Hunziker, 2021).

No existe una relación directa con la variable, si es posible un ingreso a una carrera con la educación universitaria técnica previa en el futuro sin más habilidades de TI o conocimientos de estadística. Por eso se rechaza la hipótesis H8. Sin embargo, existe una tendencia a reconocer que los auditores ven la necesidad de adaptarse.

Es interesante ver que las otras hipótesis como la digitalización en la auditoria tiene un impacto a las clases y seminarios en estudios de economía, contabilidad y administración empresas (H1), la cantidad de la información generada por la digitalización tiene un impacto a las clases y seminarios en estudios de economía, contabilidad y administración empresas (H2) y la digitalización impacta el plan de estudios de auditoría en las universidades y en la formación profesional en las empresas (H4) no tienen impacto directo a la educación de la auditoria o la formación profesional. Se puede interpretar que la necesidad de un cambio surge más por nuevas herramientas, técnicas de análisis u otras tendencias del mercado como big data, cloud computing y bases de datos que tienen directamente impacto a la educación universitaria y formación profesional en el ámbito de la auditoria. Se puede decir que las nuevas áreas de estudio son los principales factores que van a influir la educación universitaria y formación profesional para preparar los profesionales para los retos de la digitalización en la auditoria.

En resumen, se puede decir que la creciente digitalización requiere una adaptación de los conceptos de formación y perfeccionamiento para la auditoría. La encuesta también confirma el escenario de que las tareas rutinarias serán asumidas por automatismos en el futuro. Sin embargo, el auditor aún debe realizar una verificación final de los resultados. Las diferentes valoraciones de una completa toma de control de tareas exigentes por parte de los automatismos pueden explicarse por el hecho de que el primer escenario está siendo reemplazado gradualmente por el segundo escenario debido al progreso técnico.

Al resumir los resultados de la investigación, podemos concluir que los rápidos cambios en las tecnologías de la información plantean hoy la pregunta sobre las competencias de los contadores de hoy y sobre el futuro de esta profesión. Hoy en día la profesión de contador necesita un nuevo modelo capaz de dar respuesta a los cambios y desarrollos tecnológicos en el proceso de digitalización y e-transformación para que la profesión de contador sea más eficaz acorde de IFAC y el IAESB.

En la práctica internacional, la IFAC y su IAESB juegan un papel muy importante al trabajar con los problemas de los contadores profesionales. Comenzaron a trabajar activamente en 1977. El resultado de su trabajo son las Normas Internacionales de Educación para Contadores (IES). Los resultados del presente trabajan muestran que el trabajo de la organización tiene alta relevancia para la educación en México. 


\section{Referencias}

Abbott, L., Parker, S., Peters, G., \& Rama, D. (2007). Corporate Governance, Audit Quality, and the Sarbanes-Oxley Act: Evidence from Internal Audit Outsourcing. The Accounting Review, 84(4), 803-835.

Adiloglu, B., \& Gungor, N. (2019). THE IMPACT OF DIGITALIZATION ON THE AUDIT PROFESSION: A REVIEW OF TURKISH INDEPENDENT AUDIT FIRMS. Journal of Business Economics and Finance, 4, 209-214.

Alles, M. (2015). Drivers of the use and facilitators and obstacles of the evolution of big data by the audit profession. Accounting Horizons, 29(2), 439-449.

Balios, D. (2021). The Impact of Big Data on Accounting and Auditing. International Journal of Corporate Finance and Accounting, 8(1), 1-14. 
Barrados, M., \& Lonsdale, J. (2020). Opportunities, Crossover of Audit and Evaluation Practices: Challenges and Opportunities. London: Routledge.

Bhattacherjee, A. (2012). Social science research: Principles, methods, and practices. Tampa: Universtiy of South Florida: Scholar Commons.

Chin, W. W. (1998). Issues and opinions on structural equation modeling. MIS Quarterly, 22(1), 716.

Chin, W. W. (2001). PLS-Graph User's Guide Version 3.0. Soft Modeling Inc.

Cipek, K., \& Ljutić, I. (2021). THE INFLUENCE OF DIGITALIZATION ON TAX AUDIT. Oditor, 7(1), 53-69.

Clifford, C. (2019). Bill Gates: A.I. is like nuclear energy - 'both promising and dangerous'. Retrieved from CNBC. Obtenido de CNBC: https://www.cnbc.com/2019/03/26/billgatesartificial-intelligence-both-promising-and-dangerous.html

Cronbach, L. J. (1970). Essentials of Psychological Testing. Nueva York: Harper \& Row.

Falk, R., \& Miller, N. (1992). A Primer for Soft Modeling. Akron, OH: The University of Akron.

Gulin, D., Hladika, M., \& Valenta, I. (2019). Digitalization and the Challenges for the Accounting. ENTRENOVA, 502-511.

Henrizi, P., Himmlesbach, D., \& Hunziker, S. (2021). Anchoring and adjustment effects on audit judgments: experimental evidence from Switzerland. Journal of Applied Accounting Research.

Howieson, B., Hancock, P., Segal, N., Kavanagh, M., Tempone, I., \& Kent, I. (2014). Who should teach what? Australian perceptions of the roles of universities and practice in the education of professional accountants. Journal of Accounting Education, 22(1), 259-275.

IAESB. (2015). Consultation Paper, Meeting Future Expectations of Professional Competence: A Consultation on the IAESB's Future Strategy and Priorities. IFAC.

IFAC . (2018). About IFAC. Obtenido de https://www.ifac.org

Issa, H., Sun, T., \& Vasarhelyi, M. (2016). Research ideas for artificial intelligence in auditing: The formalization of audit and workforce supplementation.

Kaiser, H. (1960). The application of electronic computers to factor analysis. Educational and Psychological Measurement, 20, 141-151.

Lee, M., Cho, M., Gim, J., Jeong, D., \& Jung, H. (2014). Prescriptive analytics system for scholar research performance enhancement. International conference on human-computer interaction, 186-190.

Lind, D., Marchal, W., \& Wathen, S. (2012). Estadística aplicada a los negocios y la economía. Ciudad de México: McGrawHill.

Littley, J. (2012). Leveraging Data Analytics and Continuous Auditing Processes for Improved Audit Planning, Effectiveness, and Efficiency. . Obtenido de KPMG White Paper: http://www. kpmg.com/US/en/IssuesAndInsights/ArticlesPublications/Documents/dataanalyticscontinuous-auditing. pdf.

Manita, R., Elommal, N., Baudier, P., \& Hikkerova, L. (2020). The digital transformation of external audit and its impact on corporate governance. Technological Forecasting and Social Change, $150(\mathrm{C})$.

Maradona, A. (2020). A Qualitative Exploration of Heuristics and Cognitive Biases in Auditor Judgements. Accountability, 9(2), 94-112.

Marmolejo-Ramos, F., \& Siva Tian, T. (2010). The shifting boxplot. A boxplot based on essential summary statistics around the mean. International Journal of Psychological Research, 3(1), $37-45$.

Meuldijk, M. (2017). Impact of digitalization on the audit profession. Audit Comitee News, 33-35.

Mohajan, H. K. (2018). Qualitative research methodology in social sciences and related subjects. Journal of Economic Development, Environment and People, 7(1), 23-48. 
Narayanan, A., Bonneau, J., Felten, E., Miller, A., \& Goldfeder, S. (2016). Bitcoin and cryptocurrency technologies: a comprehensive introduction. Princeton : Princeton University Pess.

Pedrosa, I., \& Costa, C. (2012). Computer Assisted Audit Tools and Techniques in Real World: CAATT's Applications and Approaches in Context. International Journal of Computer Information Systems and Industrial Management Applications., 4, 161-168.

Plant, K., Barac, K., \& Sarens, G. (2019). Preparing work-ready graduates - skills development lessons learnt from internal audit practice. Journal of Accounting Education, 48, 33-47.

Recker, J. (2013). Scientific research in information systems: a beginner's guide. Berlin: Springer Science \& Business Media.

Schulman, J., \& Wilson, S. (2019). How blockchain technology will affect the audit. Obtenido de https://rsmus.com/what-we-do/services/assurance/howblockchain-technology-will-affect-theaudit.html

Springer Sargent, C., \& Faye Borthick, A. (2013). Evidence for Insisting on Cognitive Conflict Tasks: Impact on Accounting Majors in Upper-Level Courses. Issues in Accounting Education, 28(4), 759-777.

Stoel, D., Havelka, D., \& Merhout, J. (2012). An analysis of attributes that impact information technology audit quality: A study of IT and financial audit practitioners. International Journal of Accounting Information Systems, 13(1), 60-79.

Tekbas, I., \& Nonwoven, K. (2018). The Profession of the Digital Age: Accounting Engineering. Obtenido de http://www.ifac.org/

Tiberius, V., \& Hirth, S. (2019). Impacts of digitization on auditing: A Delphi study for Germany. Journal of International Accounting, Auditing and Taxation, 37, 1-14.

Timea Fulop, M., Tiron-Tudor, A., \& Cordos, G. (2019). Audit education role in decreasing the expectation gap. Original and Applied Research, 94(5), 306-313.

Tukey, J. (1977). Exploratory Data Analysis (Addison-Wesley Series in Behavioral Science). London: Pearson.

Vaidyanathan, N. (2017). Divided we fall, distributed we stand. Retrieved from ACCA:. Obtenido de

https://www.accaglobal.com/content/dam/ACCA_Global/Technical/Future/Divided\%20we\% 20 fall $\% 2$ C $\% 20$ distributed $\% 20 \mathrm{we} \% 20$ stand $\% 20 \% 20$ The $\% 20$ professional $\% 20$ accountant's $\% 2$ 0guide $\% 20$ to $\% 20$ distributed\%20ledgers $\% 20$ and\%20blockchain.pdf.

Vasarhelyi, M., Teeter, R., \& Krahel, J. (2010). Audit Education and the Real-Time Economy. Issues in Accounting Education, 25(3), 405-423.

Werts, C., Linn, R., \& Jöreskog, K. (1974). Interclass reliability estimates: Testing structural assumptions. Educational and Psychological Measurement, 34, 25-33.

Wessels, P. (2008). The identification and discussion of strategies for implementing an IT skills framework in the education of professional accountants. Journal South African Journal of Accounting Research, 22(1), 147-181.

Yoon, K., Hoogduin, L., \& Zhang, L. (2015). Big data as complementary audit evidence. Accounting Horizons, 29(2), 431-438.

Yordanov, V. (2018). Introduction to Natural Language Processing for Text: Retrieved from Towards Data Science . Obtenido de https://towardsdatascience.com/introductionto-naturallanguage-processing-for-text-df845750fb63 


\section{Anexos}

Tabla 1 Variables latentes, definición y rubro en el cuestionario

\begin{tabular}{|c|c|c|}
\hline \multirow{7}{*}{$\begin{array}{l}\text { Clases y seminarios } \\
\text { enfocados en } \\
\text { digitalización }\end{array}$} & Informática & 2.1 Auditoria Externa: Informática \\
\hline & Estadística & 2.2 Auditoria Externa: Estadística \\
\hline & $\begin{array}{l}\text { Desarrollo y análisis de } \\
\text { modelos de negocio }\end{array}$ & $\begin{array}{l}\text { 2.3 Auditoria Externa: Desarrollo y análisis de } \\
\text { modelos de negocio }\end{array}$ \\
\hline & Gestión de procesos & 2.4 Auditoria Externa: Gestión de procesos \\
\hline & Necesidad de cambio & $\begin{array}{l}\text { 3.1 Auditoria Externa: La formación académica } \\
\text { y profesional previa ya no será suficiente para } \\
\text { comenzar una carrera profesional en el futuro } \\
\text { como auditor o en el área del control interno de } \\
\text { una empresa: }\end{array}$ \\
\hline & $\begin{array}{l}\text { Enfoque en la clases y } \\
\text { seminarios en TI }\end{array}$ & $\begin{array}{l}\text { 3.2 Auditoria Externa: Sin una amplia } \\
\text { formación académica en TI, ya no se podrá } \\
\text { comenzar una carrera profesional en el futuro } \\
\text { como auditor o en el ámbito del control interno: }\end{array}$ \\
\hline & $\begin{array}{l}\text { Enfoque en la clases y } \\
\text { semanarios en estadística }\end{array}$ & $\begin{array}{l}\text { 3.3 Auditoria Externa: Sin una amplia } \\
\text { formación académica en estadística, ya no se } \\
\text { podrá comenzar una carrera profesional en el } \\
\text { futuro como auditor o en el ámbito del control } \\
\text { interno: }\end{array}$ \\
\hline $\begin{array}{l}\text { Impacto } \\
\text { digitalización }\end{array}$ & $\begin{array}{l}\text { Automatización de } \\
\text { actividades rutinarias }\end{array}$ & $\begin{array}{l}\text { 4. Auditoria Externa: La digitalización } \\
\text { automatizará en gran medida las tareas } \\
\text { rutinarias, de modo que el auditor externo } \\
\text { pueda concentrarse en evaluar casos especiales. }\end{array}$ \\
\hline
\end{tabular}


Automatización de tareas y actividades exigentes
5. Auditoria Externa: Los algoritmos y heurísticos altamente desarrollados, también conocidos como automatismos, asumirán tareas y actividades exigentes para las que se requiere la capacidad del juicio del auditor.
Combinación de automatización con control de calidad por humano
6. Auditoria Externa: En el futuro, el auditor externo utilizará procesos automatizados en combinación con control de calidad o control final como parte de sus actividades de auditoría:

Cambio de actividades por la 9. Auditoria Externa: La digitalización está digitalización cambiando las actividades de auditoría externa.
Cantidad de información
Aumento en la cantidad de resultados.
7. Auditoria Externa: En el futuro, el auditor externo no podrá evaluar todos los resultados de los automatismos por la gran cantidad de información que se genera. El auditor tendrá que concentrarse en muestras/ pruebas que son tanto materiales como propensas a errores.
Evaluación del entorno $\quad$ 8. Auditoria Externa: Qué opinas: La económico y legal. digitalización hace que sea mucho más difícil evaluar el entorno económico y legal, así como las oportunidades y riesgos del desarrollo futuro de una empresa:

\begin{tabular}{|c|c|c|}
\hline $\begin{array}{l}\text { Impacto en } \\
\text { recursos humanos }\end{array}$ & $\begin{array}{l}\text { Empleo de más especialistas } \\
\text { en TI y analistas. }\end{array}$ & $\begin{array}{l}\text { 10. Auditoria Externa: El departamento de } \\
\text { auditoría externa empleará a más especialistas } \\
\text { en TI y especialistas en análisis en el futuro. }\end{array}$ \\
\hline & $\begin{array}{l}\text { Impacto en puestos "Mediano } \\
\text { Plazo" }\end{array}$ & $\begin{array}{l}\text { 11.1 Auditoria Externa: ¿La digitalización } \\
\text { conducirá a la creación o reducción de puestos } \\
\text { de trabajo en auditoría externa durante los } \\
\text { próximos cinco años? }\end{array}$ \\
\hline & $\begin{array}{l}\text { Impacto en puestos "Largo } \\
\text { Plazo" }\end{array}$ & $\begin{array}{l}\text { 11.2 Auditoria Externa: ¿La digitalización } \\
\text { conducirá a un aumento o disminución de } \\
\text { puestos de trabajo en auditoría externa largo } \\
\text { plazo (más de cinco años)? }\end{array}$ \\
\hline & Perfil Empleado & $\begin{array}{l}\text { 13. Auditoria Externa: El perfil de los } \\
\text { empleados en cuanto a sus competencias en } \\
\text { auditoría externa cambiará en los próximos } \\
\text { años: }\end{array}$ \\
\hline $\begin{array}{l}\text { Impacto } \\
\text { Universidad / } \\
\text { Formación } \\
\text { profesional }\end{array}$ & Cambio plan de estudios & $\begin{array}{l}\text { 14. Auditoria Externa: El plan de estudios / } \\
\text { aprendizaje para la formación de un auditor } \\
\text { externo es actualmente demasiado extenso para } \\
\text { poder incluir nuevas áreas temáticas. }\end{array}$ \\
\hline
\end{tabular}




\begin{tabular}{lll}
\hline & $\begin{array}{l}\text { Capacitación de nuevas } \\
\text { herramientas. }\end{array}$ & $\begin{array}{l}\text { 15. Auditoria Externa: En la era de Google y } \\
\text { otras herramientas de TI, el aprendizaje de } \\
\text { procedimientos y metodologías nuevas es una } \\
\text { carga innecesaria para la formación. }\end{array}$ \\
\cline { 2 - 3 } $\begin{array}{l}\text { Capacitación en áreas } \\
\text { específicas. }\end{array}$ & $\begin{array}{l}\text { 16. Auditoria Externa: En el futuro, los } \\
\text { auditores externos deberán especializarse en } \\
\text { áreas específicas: }\end{array}$ \\
\cline { 2 - 3 } estudio & $\begin{array}{l}\text { Formación adicional (Fuera } \\
\text { de la escuela / trabajo) }\end{array}$ & $\begin{array}{l}\text { 17. Auditoria Externa: La digitalización } \\
\text { conllevara a una mayor necesidad de formación } \\
\text { adicional para los auditores externos: }\end{array}$ \\
\hline Process Mining & 12.1 Auditoria Externa: Process Mining \\
\hline Text Mining & 12.2 Auditoria Externa: Text Mining \\
\hline Automatización & 12.3 Auditoria Externa: Automatización \\
\hline Programación & 12.4 Auditoria Externa: Programación \\
\hline $\begin{array}{l}\text { Inteligencia artificial / } \\
\text { Machine Learning }\end{array}$ & $\begin{array}{l}12.5 \text { Auditoria Externa: Inteligencia artificial / } \\
\text { Machine Learning }\end{array}$ \\
\hline Blockchain & 12.6 Auditoria Externa: Blockchain \\
\hline Visualización de datos & 12.7 Auditoria Externa: Visualización de datos \\
\hline Seguridad de datos & 12.8 Auditoria Externa: Seguridad de datos \\
\hline Estadística & 12.9 Auditoria Externa: Estadística \\
\hline
\end{tabular}

Fuente: Elaboración propia.

Grafica 1 Factores que impulsan a las la educación en auditoría por la digitalización 


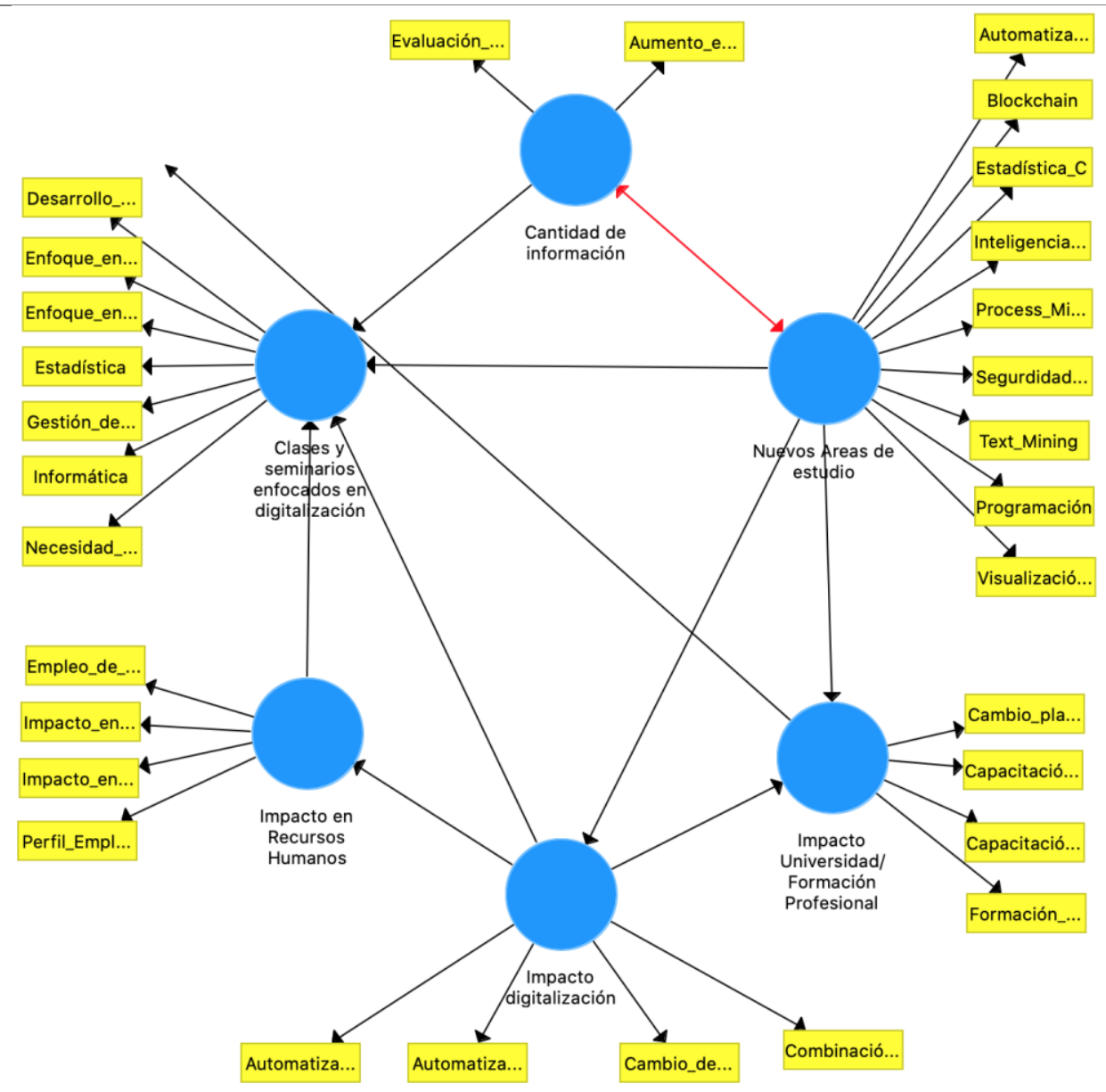

Fuente: Elaboración propia.

Grafica 2 Análisis de Alfa de Cronbach por constructo

Estadísticos de fiabilidad

\begin{tabular}{|r|r|}
\hline $\begin{array}{c}\text { Alfa de } \\
\text { Cronbach }\end{array}$ & N de elementos \\
\hline .768 & 6 \\
\hline
\end{tabular}




\begin{tabular}{|l|r|r|r|r|}
\hline & $\begin{array}{c}\text { Media de la } \\
\text { escala si se } \\
\text { elimina el } \\
\text { elemento }\end{array}$ & $\begin{array}{c}\text { Varianza de la } \\
\text { escala si se } \\
\text { elimina el } \\
\text { elemento }\end{array}$ & $\begin{array}{c}\text { Correlación } \\
\text { elemento-total } \\
\text { corregida }\end{array}$ & $\begin{array}{c}\text { Alfa de } \\
\text { Cronbach si se } \\
\text { elimina el } \\
\text { elemento }\end{array}$ \\
\hline $\begin{array}{l}\text { Clases y seminarios } \\
\text { enfocados en digitalización } \\
\text { (agrupado) }\end{array}$ & 9.94 & 5.997 & .619 & .708 \\
$\begin{array}{l}\text { Impacto digitalización } \\
\text { (agrupado) }\end{array}$ & 10.00 & 5.776 & .592 & .712 \\
$\begin{array}{l}\text { Cantidad de información } \\
\text { (agrupado) }\end{array}$ & 10.03 & 6.588 & .439 & .752 \\
$\begin{array}{l}\text { Impacto en recursos } \\
\text { humanos (agrupado) } \\
\text { Impacto Universidad } \\
\text { (agrupado) }\end{array}$ & 10.11 & 6.492 & .415 & .758 \\
$\begin{array}{l}\text { Nuevas áreas de estudio } \\
\text { (agrupado) }\end{array}$ & 10.06 & 6.009 & .549 & .747 \\
\hline Fuente: elaboración propia.
\end{tabular}

Tabla 2 Correlación de las variables latentes

\begin{tabular}{|c|c|c|c|c|c|c|}
\hline & $\begin{array}{l}\text { Cantidad de } \\
\text { información }\end{array}$ & $\begin{array}{l}\text { Clases y } \\
\text { seminarios } \\
\text { enfocados en } \\
\text { digitalización }\end{array}$ & $\begin{array}{l}\text { Impacto } \\
\text { Universidad } \\
\text { / Formación } \\
\text { profesional }\end{array}$ & $\begin{array}{l}\text { Impacto } \\
\text { digitalización }\end{array}$ & $\begin{array}{l}\text { Impacto } \\
\text { en } \\
\text { recursos } \\
\text { humanos }\end{array}$ & $\begin{array}{l}\text { Nuevas } \\
\text { ares de } \\
\text { estudio }\end{array}$ \\
\hline Cantidad de información & 0.691 & & & & & \\
\hline $\begin{array}{l}\text { Clases y seminarios } \\
\text { enfocados en digitalización }\end{array}$ & 0.416 & 0.632 & & & & \\
\hline $\begin{array}{l}\text { Impacto Universidad / } \\
\text { Formación profesional }\end{array}$ & 0.427 & 0.343 & 0.657 & & & \\
\hline
\end{tabular}




\begin{tabular}{lccccc}
\hline Impacto digitalización & 0.314 & 0.531 & 0.44 & $\mathbf{0 . 6 5 1}$ & \\
\hline $\begin{array}{l}\text { Impacto en recursos } \\
\text { humanos }\end{array}$ & 0.078 & 0.29 & 0.568 & 0.467 & $\mathbf{0 . 6 7 8}$ \\
\hline Nuevas ares de estudio & 0.34 & 0.695 & 0.44 & 0.623 & 0.456 \\
\hline Nivel de significancia de 0.05 & & & & & $\mathbf{0 . 6 4 5}$ \\
\hline Fuente: Elaboración propia. & & & &
\end{tabular}

Tabla 3 Resultados del modelo estructural. Relaciones entre constructos. [N=108]

\begin{tabular}{|c|c|c|c|c|c|}
\hline & & & & $\boldsymbol{\beta}$ & valor $\mathbf{t}$ \\
\hline H1 & Impacto digitalización & $\rightarrow$ & $\begin{array}{l}\text { Clases y seminarios } \\
\text { enfocados en digitalización }\end{array}$ & 0.149 & 1.402 \\
\hline H2 & Cantidad de información & $\rightarrow$ & $\begin{array}{l}\text { Clases y seminarios } \\
\text { enfocados en digitalización }\end{array}$ & 0.18 & 1.923 \\
\hline H3 & Impacto digitalización & $\rightarrow$ & Impacto en recursos humanos & 0.46 & 5.065 \\
\hline H4 & Impacto digitalización & $\rightarrow$ & $\begin{array}{l}\text { Impacto Universidad / } \\
\text { Formación profesional }\end{array}$ & 0.264 & 2.158 \\
\hline H5 & Nuevas ares de estudio & $\rightarrow$ & $\begin{array}{l}\text { Impacto Universidad / } \\
\text { Formación profesional }\end{array}$ & 0.276 & 2.369 \\
\hline H6 & Nuevas ares de estudio & $\rightarrow$ & $\begin{array}{l}\text { Clases y seminarios } \\
\text { enfocados en digitalización }\end{array}$ & 0.564 & 4.161 \\
\hline H7 & Cantidad de información & $\rightarrow$ & Nuevas ares de estudio & 0.34 & 3.562 \\
\hline H8 & Impacto en recursos humanos & $\rightarrow$ & $\begin{array}{l}\text { Clases y seminarios } \\
\text { enfocados en digitalización }\end{array}$ & -0.05 & 0.319 \\
\hline H9 & Nuevas ares de estudio & $\rightarrow$ & Impacto digitalización & 0.58 & 9.892 \\
\hline H10 & Cantidad de información & $\rightarrow$ & Impacto digitalización & 0.124 & 1.52 \\
\hline
\end{tabular}

Fuente: Elaboración propia.

Tabla 4: R Cuadrada

\begin{tabular}{ll}
\hline & R Cuadrada \\
\hline Cantidad de información & 0.321 \\
\hline $\begin{array}{l}\text { Clases y seminarios enfocados en } \\
\text { digitalización }\end{array}$ & 0.533 \\
\hline $\begin{array}{l}\text { Impacto Universidad / } \\
\text { Formación profesional }\end{array}$ & 0.237 \\
\hline Impacto digitalización & 0.401 \\
\hline Impacto en recursos humanos & 0.212 \\
\hline
\end{tabular}


Nuevas ares de estudio

0.115

Nivel de significancia de 0.05

Fuente: Elaboración propia. 